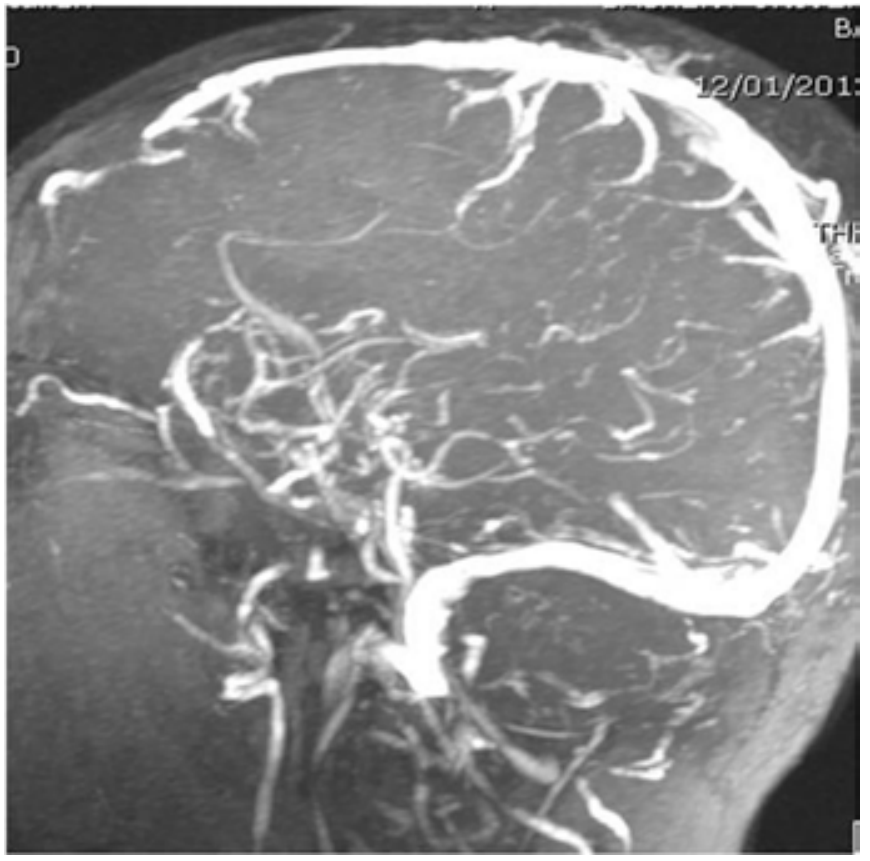

Abstract 524 Figure 2a

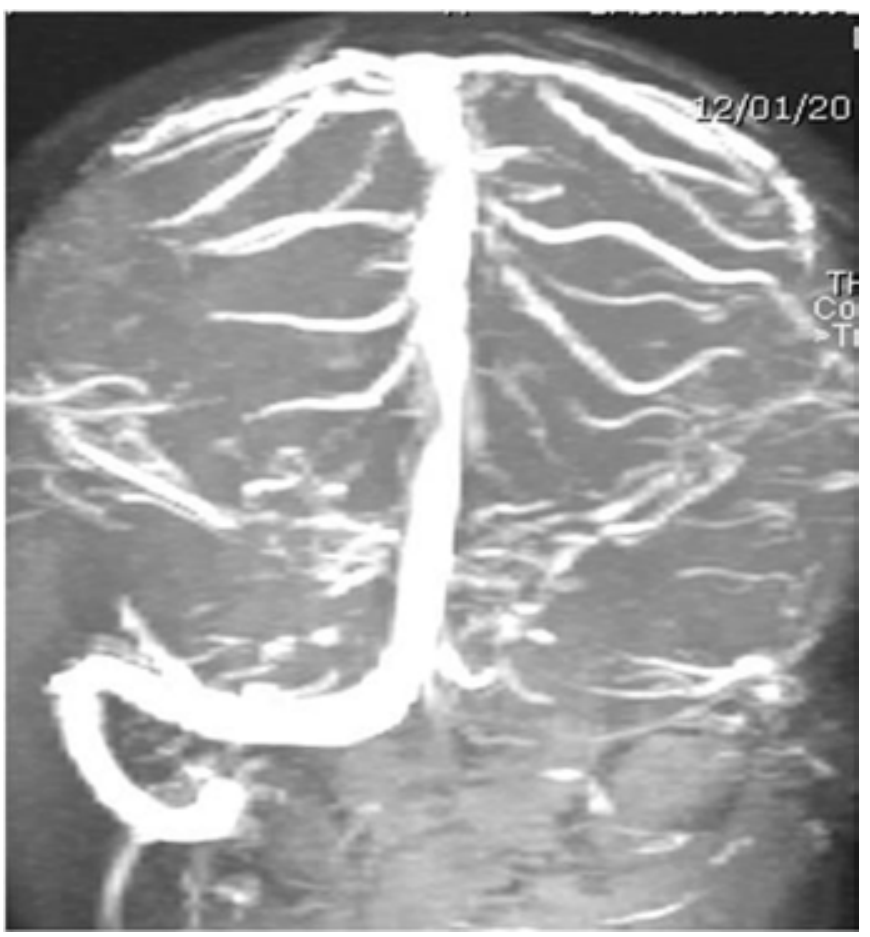

Abstract 524 Figure 2b

Evaluation for thrombophilia and tests for specific predisposing conditions were normal. The patient was treated by third generation cephalosporin, clindamycin and low-molecular weight heparin. Two months after the initial diagnosis, the evidence of a small focal thalamic hemorrhage was noted on MRI. Recanalization of venous occlusions were detected on MRI venography.Low-molecular weight heparin therapy was changed with oral acetylsalicylic acid.

Conclusion Since CSVT is an underrecognized cause of intracranial pathologic abnormality in the pediatric population, we wish to draw the attention of pediatricians to the importance of a high level of suspicion for this diagnosis and the preferred diagnostic method which is MRI with venography.

\section{PAEDIATRIC MOYAMOYA SYNDROME ASSOCIATED WITH IRON DEFICIENCY ANAEMIA: A CASE REPORT AND REVIEW OF THE LITERATURE}

doi:10.1136/archdischild-2012-302724.0525

K McElroy, A Kennedy, L Houliston, A Judkins, G Chaney, P Richmond. Department of Paediatric and Adolescent Medicine, Princess Margaret Hospital for Children, Perth, WA, Australia

Introduction Moyamoya syndrome is a rare, progressive disorder characterised by stenosis of the vasculature of the Circle of Willis with formation of a tortuous collateral system. The aetiology may be idiopathic, or as a consequence of a number of associated diseases. No previous cases secondary to iron-deficiency anaemia have been reported.

Aim To report a case of Moyamoya syndrome secondary to irondeficiency anaemia, and review the literature with regard to the haematological associations of Moyamoya syndrome.

Method The relevant case notes were reviewed. Key databases were searched for studies including the target phrases "Moyamoya syndrome" and "anaemia".

Results Case history: A 3 year old girl presents with a 12 hour history of right upper limb weakness and slurred speech. A background of pallor, daily vomiting and abdominal pain is elicited. Reduced power in the right upper and lower limbs and a right-sided upgoing plantar are noted on examination. Investigations reveal a haemoglobin of $1.9 \mathrm{~g} / \mathrm{dL}$, with an iron-deficiency pattern. A faecal occult blood test is positive. Magnetic Resonance (MR) imaging shows focal middle cerebral artery territory ischaemia. MR Angiography shows Moyamoya vessels. Extensive investigations for associated pathologies are normal.

Literature review: Sickle cell disease is the most common haematological cause of the Moyamoya syndrome, whilst thalassemia, Fanconi's anaemia and paroxysmal nocturnal haemoglobinuria are also reported. The likely pathogenesis in these instances is intimal proliferation in response to impaired arterial flow.

Conclusion We propose that severe iron-deficiency anaemia may result in Moyamoya syndrome through the mechanism of disrupted intracerebral arterial flow.

\section{PROTOCOLS FOR THE TREATMENT OF HODGKIN LYMPHOMA - CASE REPORT}

doi:10.1136/archdischild-2012-302724.0526

Al Krasniqi, V Grajqevci-Uka, R Maqastena-Maxhuni, B Abrashi, F Selimi, E Bajrami, E Islamaj, S Nushi. Hemato-Oncology Department, UCCK, Pediatric Clinic, Prishtina, Kosovo

Introduction Hodgkin lymphoma is malignant disease of cells in the lymphatic system that is characterized by proliferation of Reed Sternberg cells. Symptoms include the painless swelling of lymph nodes, spleen, or other immune tissue. Other symptoms include fever, weight loss, fatigue, or night sweats. Also called Hodgkin disease.

Objective Was the presentation a case with Hodgkin lymphoma, treated in Pediatric Clinic in Prishtina at the Hemato-Oncology Unit.

Methods Patient, male child, 9 years old, came in the clinic because of painless enlargement of lymph nodes on the left side of the neck. Other examinations, according to systems have been in normal range. Diagnosis is made based on anamnesis, clinical examination, laboratory, ultrasound, CT, histopathology with immunochemistry, and bone marrow aspiration. The surgery has been made and according to path-histological and immunochemistry analyses the 